\title{
LEARNING IN DISORDERS OF CONSCIOUS
}

Assessing awareness in patients with disorders of consciousness (DOCs) is difficult, as clinicians cannot rely on explicit reports. Using a simple classical conditioning paradigm, Bekinschtein et al. have now demonstrated learning in patients with DOCs, indicating some preservation of conscious processing. The extent of learning also correlated with the severity of injury and was a good indicator of recovery.

The term 'DOCs' encompasses a spectrum of individuals with severe brain damage, but clinical differentiation within this group can be challenging. Classification is based on patients' external awareness; patients in a vegetative state demonstrate no awareness, whereas those in the minimally conscious state show some inconsistent awareness and, if they improve and start to communicate, may be reclassified as severely disabled.

Objective tests of awareness in patients with DOCs cannot rely on explicit reports as do traditional tests. Trace conditioning of the eyeblink response, a simple form of associative learning, was identified as a potentially useful measure of learning by the researchers. In this paradigm, a tone is presented $500 \mathrm{~ms}$ before a puff of air to the eye in a series of training trials, leading to development of a conditioned blink response. Awareness of the temporal contingency between stimuli is necessary to learn this association.

Bekinschtein et al. trained a heterogeneous DOC group, as well as conscious and unconscious (anesthetized with propofol) control groups, with the above paradigm. Normalized electromyographic (nEMG) scans provided a measure of the magnitude and specificity of learning by tracking event-related responses in the anticipatory interval between presentation of the tone and the air puff. The researchers found that $\mathrm{nEMG}$ responses increased in the conscious control and DOC groups during this interval, indicating that they had learned the association. The extent of this learning was "significant, though considerably less prominent, in the DOC group", according to the researchers. These results suggest the presence of partially preserved conscious processing in DOCs, and show this paradigm to be a good objective test of this phenomenon.

An alternative interpretation of these data is that trace conditioning occurs in the absence of consciousness, and so is not an indicator of awareness. However, the unconscious group's failure to learn suggests that this is not the case. Furthermore, subsequent follow-up of the patients with DOCs showed that learning in the trace conditioning task predicts "with an accuracy of $86 \%$, whether a subject has shown signs of recovery or not." In all the patients who showed recovery, the degree of brain atrophy was a good predictor of learning capabilities. These results can, therefore, be taken to more strongly indicate that "patients retained some covert conscious processing," according to Bekinschtein.

Trace conditioning is a promising tool for classifying DOCs and a potential indicator of recovery. Bekinschtein hopes that "this paradigm [can be used] as a simple bedside test to assess learning and conscious processing," although this should be part of a broader battery of other "anatomical, functional and behavioral measures." The researchers speculate that "training the circuits involved in awareness may help the recovery of consciousness."

Eleanor Beal

Original article Bekinschtein, T. A. et al. Classical conditioning in the vegetative and minimally conscious state. Nat. Neurosci. 12, 1343-1349 (2009). 